\title{
A New Structure on Manifolds: Silver Structure
}

\author{
Mustafa Özkan* and Betül Peltek
}

(Communicated by Josef Mikeš)

\begin{abstract}
In this paper, we address a new structure defined by a $(1,1)$ tensor field $\Theta$ satisfying $\Theta^{2}-2 \Theta-I=0$ on a manifold, which is called silver structure. Integrability conditions and parallelism of the this structure is obtained via a corresponding almost product structure. Finally, a silver Riemannian structure is defined on a Riemannian manifold.
\end{abstract}

Keywords: Almost product structure; silver structure; integrability; silver Riemannian manifold; Pell sequence.

AMS Subject Classification (2010): Primary: 53C15.

\section{Introduction}

Irrational numbers have an impact as fascinating as rational numbers. One of such fascinating impacts that attracted the attention most is the golden ratio $\phi=(1+\sqrt{5}) / 2$ number, which is a positive root of the equation $x^{2}-x-1=0$. Inspired by this algebraic equation, Hrețcanu [13] defined the golden structure on a manifold $M$ by a tensor field $\Phi$ of type $(1,1)$ on $M$ satisfying $\Phi^{2}=\Phi+I$. Then, the geometry of the golden structure on $M$ was investigated by Crasmareanu and Hreţcanu [3]. Recently, the golden structure has been studied in $[6,7,14,15,22,24,25,26,28,29,32]$. In addition to these, some types of polynomial structures was intensively studied in last time, namely an almost product, almost complex, almost tangent and $f$-structure in $[9,10,11,17,18,19]$.

Another irrational number, as fascinating as the golden ratio, is $\theta=1+\sqrt{2}$, which is a positive solution of the equation $x^{2}-2 x-1=0$ and called the silver number, silver ratio, or silver mean. It has been used in design, architecture, and physics. Also, Chandra and Rani [2] used the silver mean to describe fractal geometry.

The main novelty of this paper is to study the geometry of the silver structure on a differentiable manifold using a corresponding almost product structure. To the best of our knowledge, it is the first time that silver structure on manifolds is studied in the literature. In particular, we follow the spirit of [3].

The paper is organized as follows. In Section 2, we define silver structure on a differentiable manifold. Furthermore, we establish the relationship among the silver ratio, tangent real silver ratio, and complex silver ratio. Next, we give some examples of silver structure. After that, we study the connection on the silver structure. In Section 5 we investigate the integrability of the silver structure, and the parallelism of the silver structure in terms of Schouten and Vrănceanu connections. Finally, we define silver Riemannian manifold and study some properties on this manifold. Also, we give an example of the silver structure on manifold $\mathbb{R}^{2}$.

\section{Silver Structures on Manifolds}

Throughout the paper, all manifolds, tensor fields, and connections are assumed to be differentiable and of class $C^{\infty}$.

Now, we give some definitions and propositions that will be used in the rest of this paper. 
Definition 2.1 (see [23]). Let $M$ be a $C^{\infty}$ differentiable manifold. A tensor field $\Theta$ of type $(1,1)$ on $M$ is called a silver structure on $M$ if $\Theta$ satisfies the equation

$$
\Theta^{2}=2 \Theta+I
$$

where $I$ is the identity $(1,1)$ tensor field on $M$.

Proposition 2.1 (see [20]). Let $\Theta$ be a silver structure on the manifold $M$. For any integer number $n$

$$
\Theta^{n}=P_{n} \Theta+P_{n-1} I
$$

where $\left(P_{n}\right)$ is the Pell sequence.

Using the Binet's formula for Pell sequence $[12,16]$ which is

$$
P_{n}=\frac{\theta^{n}-(2-\theta)^{n}}{2 \sqrt{2}}
$$

from (2.2) we have

$$
\Theta^{n}=\frac{\theta^{n}-(2-\theta)^{n}}{2 \sqrt{2}} \Theta+\frac{\theta^{n-1}-(2-\theta)^{n-1}}{2 \sqrt{2}} I .
$$

Proposition 2.2. $\quad$ i) The eigenvalues of a silver structure $\Theta$ are the silver ratio $\theta$ and $2-\theta$.

ii) A silver structure $\Theta$ is an isomorphism on the tangent space $T_{x} M$ for every $x \in M$ of the manifold $M$.

iii) $\Theta$ is invertible and its inverse $\hat{\Theta}=\Theta^{-1}$ satisfies:

$$
\hat{\Theta}^{2}=-2 \hat{\Theta}+I .
$$

From the paper [3] if $T, P$, and $J$ are an almost tangent structure, an almost product structure, and an almost complex structure, respectively, then $-T,-P$ and $-J$ are also an almost tangent structure, an almost product structure, and an almost complex structure, respectively. One should emphasize that we can find a similar relation in a silver structure:

Proposition 2.3 (see [23]). If $\Theta$ is a silver structure then $\tilde{\Theta}=2 I-\Theta$ is also a silver structure.

One can easily see from the following assertion that it is clear to obtain a connection between a silver and almost product structure.

Theorem 2.1. If $\Theta$ is a silver structure on $M$, then

$$
P=\frac{1}{\sqrt{2}}(\Theta-I)
$$

is an almost product structure on $M$. Conversely, any almost product structure $P$ on $M$ yields a silver structure on $M$ as follows:

$$
\Theta=I+\sqrt{2} P .
$$

By the equation (2.4), we can give following definitions.

Definition 2.2. 1) Let $(M, T)$ be an almost tangent manifold. The tensor field $\Theta_{t}$ defined by

$$
\Theta_{t}=I+\sqrt{2} T
$$

is called the tangent silver structure on $(M, T) . \Theta_{t}$ satisfies the equation

$$
\Theta_{t}^{2}-2 \Theta_{t}+I=0 .
$$

Taking into account the equation $x^{2}-2 x+1=0$ in the real field $\mathbb{R}$, we have the tangent real silver ratio $\theta_{t}=1$. 
2) Let $(M, J)$ be an almost complex manifold. The tensor field $\Theta_{c}$ defined by

$$
\Theta_{c}=I+\sqrt{2} J
$$

is called the complex silver structure on $(M, J)$. The polynomial equation satisfied by $\Theta_{c}$ is

$$
\Theta_{c}^{2}-2 \Theta_{c}+3 I=0 .
$$

For $M=\mathbb{R}$ we get

$$
x^{2}-2 x+3=0
$$

with solutions $x_{1}=1+i \sqrt{2}, x_{2}=1-i \sqrt{2}$. The complex number $\theta_{c}=1+i \sqrt{2}$ is called a complex silver ratio.

We give the relationship among the silver ratio, tangent real silver ratio, and complex silver ratio as follows:

$$
\begin{array}{ccc}
\text { Tangent real silver ratio } & \text { Silver ratio } & \text { Complex silver ratio } \\
\theta_{t}=1 & \theta=1+\sqrt{2} & \theta_{c}=1+i \sqrt{2} \\
& =\theta_{t}+i\left(\theta-\theta_{t}\right)
\end{array}
$$

\section{Examples of Silver Structure}

In this part of the paper, we give some silver structure examples.

Example 3.1 (Clifford Algebras). Let $C^{\prime}(n)$ be the real Clifford algebra of the positive definite form $\sum_{i=1}^{n}\left(x^{i}\right)^{2}$ of $\mathbb{R}^{n}$ [21]. The defining relations of $C^{\prime}(n)$ are

$$
\left\{\begin{array}{l}
e_{i}^{2}=1 \\
e_{i} e_{j}+e_{j} e_{i}=0, \quad i \neq j
\end{array}\right.
$$

where $\left\{e_{1}, \ldots, e_{n}\right\}$ is orthonormal basis of $\mathbb{R}^{n}$.

Therefore, introducing $\Theta_{i}=1+\sqrt{2} e_{i}$ we give new presentation relations of $C^{\prime}(n)$ :

$$
\left\{\begin{array}{l}
\Theta_{i}: \text { Silver structure } \\
\Theta_{i} \Theta_{j}+\Theta_{j} \Theta_{i}=2\left(\Theta_{i}+\Theta_{j}\right)-2, i \neq j .
\end{array}\right.
$$

In [21], $C^{\prime}(2)$ is constructed as

$$
1=I_{2}, \quad e_{1}=\left(\begin{array}{cc}
1 & 0 \\
0 & -1
\end{array}\right), e_{2}=\left(\begin{array}{ll}
0 & 1 \\
1 & 0
\end{array}\right)
$$

and hence

$$
\left\{\begin{aligned}
& \text { i) } \Theta_{1}=I_{2}+\sqrt{2} e_{1}=\left(\begin{array}{cc}
1+\sqrt{2} & 0 \\
0 & 1-\sqrt{2}
\end{array}\right)=\left(\begin{array}{cc}
\theta & 0 \\
0 & 2-\theta
\end{array}\right), \\
& \text { ii) } \Theta_{2}=I_{2}+\sqrt{2} e_{2}=\left(\begin{array}{cc}
1 & \sqrt{2} \\
\sqrt{2} & 1
\end{array}\right) .
\end{aligned}\right.
$$

Example 3.2 (2D Silver Matrices). $\Theta \in \mathbb{R}_{n}^{n}$ is called a silver matrix if $\Theta$ satisfies the equation

$$
\Theta^{2}=2 \Theta+I_{n}
$$

where $I_{n}$ is the identity matrix on $\mathbb{R}_{n}^{n}$.

By solving (3.2) for $n=2$, we obtain silver structures in $\mathbb{R}_{2}^{2}$;

i) For $a, d \in \mathbb{R}, b \in \mathbb{R}-\{0\}$,

$$
\Theta_{a, b}=\left(\begin{array}{cc}
a & -\frac{1}{b}\left(a^{2}-2 a-1\right) \\
b & 2-a
\end{array}\right) \text { or } \Theta_{b, d}=\left(\begin{array}{cc}
2-d & -\frac{1}{b}\left(d^{2}-2 d-1\right) \\
b & d
\end{array}\right) .
$$


ii) For $a=\theta, b \in \mathbb{R}$,

$$
\begin{aligned}
\Theta_{\theta, b} & =\left(\begin{array}{cc}
\theta & 0 \\
b & 2-\theta
\end{array}\right) \text { or } \Theta_{2-\theta, b}=\left(\begin{array}{cc}
2-\theta & 0 \\
b & \theta
\end{array}\right) \text { or } \\
\Theta_{\theta, b} & =\left(\begin{array}{cc}
\theta & b \\
0 & 2-\theta
\end{array}\right) \text { or } \Theta_{2-\theta, b}=\left(\begin{array}{cc}
2-\theta & b \\
0 & \theta
\end{array}\right) .
\end{aligned}
$$

iii) For $a=\theta, b=0$,

$$
\Theta_{\theta, 0}=\left(\begin{array}{cc}
\theta & 0 \\
0 & 2-\theta
\end{array}\right) \text { or } \Theta_{2-\theta, 0}=\left(\begin{array}{cc}
2-\theta & 0 \\
0 & \theta
\end{array}\right)
$$

Hence, from (3.1) and (3.3) we have

$$
\Theta_{1}=\lim _{b \rightarrow 0} \Theta_{\theta, b} \quad \text { and } \quad \Theta_{2}=\Theta_{1, \sqrt{2}} .
$$

Example 3.3 (Quaternion Algebras). Let $\mathbb{H}$ be a quaternion algebra with a base $\left\{1, e_{1}, e_{2}, e_{3}\right\}$ satisfying

$$
e_{1}^{2}=e_{2}^{2}=e_{3}^{2}=-1, e_{1} e_{2}=-e_{2} e_{1}=e_{3}, e_{2} e_{3}=-e_{3} e_{2}=e_{1}, e_{3} e_{1}=-e_{1} e_{3}=e_{2} .
$$

We can write any quaternion as follows

$$
q=S_{q}+\vec{V}_{q}=a_{0}+a_{1} e_{1}+a_{2} e_{2}+a_{3} e_{3}
$$

where $S_{q}=a_{0}$ and $\vec{V}_{q}=a_{1} e_{1}+a_{2} e_{2}+a_{3} e_{3}$ denote the scalar and vector parts of $q$, respectively.

The norm of a quaternion $q$ is defined by $N_{q}=\sqrt{a_{0}^{2}+a_{1}^{2}+a_{2}^{2}+a_{3}^{2}}$ and we say that $q_{0}=q / N_{q}$ is a unit quaternion where $q \neq 0$. Every unit quaternion can be written in the form: $q_{0}=\cos \alpha+\vec{\varepsilon}_{0} \sin \alpha$ where $\vec{\varepsilon}_{0}$ is a unit vector satisfying the equality $\vec{\varepsilon}_{0}^{2}=-1$.

Hence;

i) We can define the silver biquaternion structure as

$$
\Theta_{q}=1+\sqrt{2} i \vec{\varepsilon}_{0}
$$

where $\vec{\varepsilon}_{0}^{2}=-1$ and $i^{2}=-1$.

ii) We can define the silver split quaternion structure as

$$
\Theta_{q}=1+\sqrt{2} \vec{\varepsilon}_{0}
$$

where $\vec{\varepsilon}_{0}^{2}=1$.

Example 3.4 (Silver Reflection). Recall, $[3,21]$, that in an Euclidean space $(E,<,>)$, the reflection with respect to a hyperplane $H$ with the normal $v \in E-\{0\}$ has the formula

$$
r_{v}(x)=x-\frac{2\langle x, v>}{<v, v>} v \text { for } x \in E
$$

and obviously $r_{v}^{2}=I_{E}$ the identity on $E$.

Hence, we can define the silver reflection with respect to $v$ as

$$
\Theta_{v}=I_{E}+\sqrt{2} r_{v}
$$

and then $v$ is an eigenvector of $\Theta_{v}$ with the corresponding eigenvalue $2-\theta$. Also, the Lemma from [21, p.314] follows that,

$$
X \Theta_{v} X^{-1}=\Theta_{X(v)}
$$

for $X \in O(E,<,>)$ : the orthogonal group of $E$. An explicit expression of this linear transformation is

$$
\Theta_{v}(x)=\theta x-2 \sqrt{2} \frac{<x, v>}{<v, v>} v .
$$

Example 3.5 (Triple Structure in Terms of Silver Structures). Let $F$ and $P$ be two tensor fields of type $(1,1)$ on the manifold $M$. With the triple $(F, P, J=P \circ F)$ we can define the following four structures: 
1) $F^{2}=P^{2}=I$ and $P \circ F-F \circ P=0$; then $J^{2}=I$,

2) $F^{2}=P^{2}=I$ and $P \circ F+F \circ P=0$; then $J^{2}=-I$,

3) $F^{2}=P^{2}=-I$ and $P \circ F-F \circ P=0$; then $J^{2}=I$,

4) $F^{2}=P^{2}=-I$ and $P \circ F+F \circ P=0$; then $J^{2}=-I$

called, respectively, almost hyperproduct (ahp), almost biproduct complex (abpc), almost product bicomplex $(\mathrm{apbc})$, and almost hypercomplex (ahc) [3,4].

From (2.4), we can write

$$
\Theta_{F}=I+\sqrt{2} F, \quad \Theta_{P}=I+\sqrt{2} P, \quad \Theta_{J}=I+\sqrt{2} J
$$

where $F, P$ tensor fields of type $(1,1)$ on the manifold $M$ and $J=P \circ F$. Hence we get

$$
\sqrt{2} \Theta_{J}=\Theta_{P} \Theta_{F}-\Theta_{p}-\Theta_{F}+\theta I
$$

and the triple $\left(\Theta_{F}, \Theta_{P}, \Theta_{J}\right)$ is:

$\left.1^{\prime}\right)$ An (ahp)-structure if and only if $\Theta_{F}, \Theta_{P}$ are silver structures and $\Theta_{P} \Theta_{F}=\Theta_{F} \Theta_{P}$ then $\Theta_{J}$ is a silver structure .

$\left.2^{\prime}\right)$ An (abpc)-structure if and only if $\Theta_{F}, \Theta_{P}$ are silver structures and $\Theta_{P} \Theta_{F}+\Theta_{F} \Theta_{P}=2\left(\Theta_{P}+\Theta_{F}\right)-2 I$ then $\Theta_{J}$ is a complex silver structure.

$\left.3^{\prime}\right)$ An (apbc)-structure if and only if $\Theta_{F}, \Theta_{P}$ are complex silver structures and $\Theta_{P} \Theta_{F}=\Theta_{F} \Theta_{P}$ then $\Theta_{J}$ is a silver structure.

$\left.4^{\prime}\right)$ An (ahc)-structure if and only if $\Theta_{F}, \Theta_{P}$ are complex silver structures and $\Theta_{P} \Theta_{F}+\Theta_{F} \Theta_{P}=2\left(\Theta_{P}+\right.$ $\left.\Theta_{F}\right)-2 I$ then $\Theta_{J}$ is a complex silver structure.

\section{Connection as Silver Structure}

\subsection{Connections in principal fibre bundles}

Let $P(M, \pi, G)$ be a principal fibre bundle with total space $P$, base space $M$, projection $\pi$, and structure group $G$. $V$ and $H$ denote the vertical distribution (the kernels of $\pi_{*}$ ) and the horizontal distribution (complementary distribution, i.e. $T P=V \oplus H$ and $H$ is $G$-invariant), respectively.

The tensor field of type $(1,1)$

$$
F=v-h
$$

is an almost product structure on $P$ where $v$ and $h$ are the corresponding projectors of $V$ and $H$, respectively.

We know in [3] that, $F$ represents a principal connection if and only if the following conditions are satisfied:

i) $X$ is a vertical vector field if and only if $F(X)=X$.

ii) $d R_{a} \circ F_{u}=F_{u a} \circ d R_{a}$ for every $a \in G$ and $u \in P$.

Taking into account (2.4), we have the following assertions for a silver structure.

Proposition 4.1. Let $\Theta$ be a silver structure on $P . \Theta$ is associated to a principal connection if and only if the following relations hold:

i) $X \in V$ if and only if $X \in \chi(P)$ is an eigenvector of $\Theta$ with respect to the eigenvalue $\theta$.

ii) $d R_{a} \circ \Theta_{u}=\Theta_{u a} \circ d R_{a}$ for every $a \in G$ and $u \in P$.

Let $\omega \in \Omega^{1}(P, g)$ be the connection 1 -form of $H$ and let $\Omega \in \Omega^{2}(P, g)$ be the curvature form of $\omega$ where $g$ is the Lie algebra of $G$. We have [3]

$$
\Omega(X, Y)=-\frac{1}{4} \omega\left(N_{F}(X, Y)\right)
$$

where $N_{F}$ is the Nijenhuis tensor of $F$. 
Proposition 4.2. Let $F$ be an almost product structure and let $\Theta$ be the associated (2.4) silver structure. Then

$$
N_{F}=\frac{1}{2} N_{\Theta}
$$

and

$$
\Omega(X, Y)=-\frac{1}{8} \omega\left(N_{\Theta}(X, Y)\right)
$$

where $N_{F}$ and $N_{\Theta}$ are the Nijenhuis tensors of $F$ and $\Theta$, respectively.

We know that the principal connection is flat if its curvature form $\Omega$ vanishes.

Proposition 4.3. The principal connection is flat if and only if the associated silver structure is integrable, i.e. $N_{\Theta}=0$.

The given principal connection determines a lift $l_{\omega}: \chi(M) \rightarrow \chi(P)$ satisfying

$$
\left[l_{\omega} \bar{X}, l_{\omega} \bar{Y}\right]-l_{\omega}[\bar{X}, \bar{Y}]=N_{F}\left(l_{\omega} \bar{X}, l_{\omega} \bar{Y}\right)
$$

for $\bar{X}, \bar{Y} \in \chi(M)[3]$.

Proposition 4.4. The lift $l_{\omega}$ is a morphism if and only if the associated silver structure is integrable, i.e. $N_{\Theta}=0$.

\subsection{Connection in tangent bundles}

Let $M$ be an $n$-dimensional differentiable manifold and $\left(T M, \pi_{M}, M\right)$ is its tangent bundle with the base space $M$. Let $\left(U, x^{i}\right)_{1 \leq i \leq n}$ be a local coordinate system on $M$ and $\left(\pi_{M}^{-1}(U), x^{i}, y^{i}\right)_{1 \leq i \leq n}$ be induced local coordinate system on $T M$ defined by $x^{i}(u)=x^{i}\left(\pi_{M}(u)\right)$ and $y^{i}(u)=d x^{i}(u)$ for all $u \in \pi_{M}^{-1}(U) . V=$ $\left\{X \in T M: \pi_{M *}(X)=0\right\}$ called the vertical distribution of $M$.

A tensor field $T$ of type $(1,1)$ on $M$ defined by $T=\frac{\partial}{\partial y^{i}} \otimes d x^{i}$ an almost tangent structure on $M$, i.e. $T^{2}=0$.

Definition 4.1 (see [3]). A $(1,1)$ tensor field $v$ is called vertical projector if

$$
T \circ v=0, \quad v \circ T=T .
$$

Definition 4.2 (see [3]). A complementary distribution $N$ to the $V$

$$
\chi(M)=N \oplus V,
$$

is called non-linear connection.

Since a vertical projector $v$ is $C^{\infty}(M)$ linear with $i m v=V$, we obtain

Proposition 4.5 (see [3]). A vertical vector $v$ induces a non-linear connection denoted $N(v)$ through relation $N(v)=$ ker $v$.

If $N$ is a non-linear connection then $h_{N}$ and $v_{N}$ are the horizontal and vertical projection with respect to the decomposition (4.1). Thus we have

Proposition 4.6 (see [3]). Let $h_{N}$ and $v_{N}$ corresponding projections $N$ and $V$, respectively. If $N$ is a non-linear connection then $v_{N}$ is a vertical projector with $N\left(v_{N}\right)=N$.

Definition 4.3 (see [3]). A $(1,1)$ tensor field $\Gamma$ is called non-linear connection of an almost product type if

$$
\Gamma \circ T=-T, \quad T \circ \Gamma=T .
$$

Proposition 4.7 (see [3]). If $\Gamma$ is a non-linear connection of an almost product type, then

i) $v_{\Gamma}=\frac{1}{2}\left(I_{\chi(M)}-\Gamma\right)$ is a vertical vector.

ii) $V(M)$ is the $(-1)$-eigenspace of $\Gamma$ while $N\left(v_{\Gamma}\right)$ is the $(+1)$-eigenspace of $\Gamma$.

Proposition 4.8 (see [3]). Let $\Gamma=I_{\chi(M)}-2 v$ be a non-linear connection of an almost product type where $v$ is a vertical vector. Then $\Gamma$ is an almost product structure on $M$.

The following proposition with regard to silver structures is posed:

Proposition 4.9. A non-linear connection $N$ on $M$, given by the vertical vector $v$, can also be defined by a silver structure $\Theta\left(=\Theta_{\Gamma}\right)$

$$
\Theta=\theta I_{\chi(M)}-2 \sqrt{2} v
$$

with $N$ the $\theta$-eigenspace and $V$ the $(2-\theta)$-eigenspace. 


\section{Integrability and Parallelism of Silver Structures}

Let $\Theta$ be a silver structure on $M . N_{\Theta}$ denotes the Nijenhuis tensor of tensor field $\Theta$ of type (1,2). From [30], for $X, Y \in \chi(M)$

$$
N_{\Theta}(X, Y)=\Theta^{2}[X, Y]+[\Theta X, \Theta Y]-\Theta[\Theta X, Y]-\Theta[X, \Theta Y] .
$$

$R, S$ denote the complementary distributions on $M$ corresponding to $\theta$ and $2-\theta$, respectively. Let $r, s$ be the corresponding projections, which results in

$$
r^{2}=r, s^{2}=s, r s=s r=0, r+s=I .
$$

Based on the straightforward computation from (2.4), we have the following equations

$$
\left\{\begin{array}{l}
r=\frac{1}{2 \sqrt{2}} \Theta-\frac{2-\theta}{2 \sqrt{2}} I \\
s=-\frac{1}{2 \sqrt{2}} \Theta+\frac{\theta}{2 \sqrt{2}} I .
\end{array}\right.
$$

For convenience of the reader, we give the next theorem for integrability of $\Theta$, the distribution $R$ and $S$. We know from [30] that

i) $\Theta$ is integrable if $N_{\Theta}=0$.

ii) The distribution $R$ is integrable if $s[r X, r Y]=0$ and $S$ is integrable if $r[s X, s Y]=0$ for $X, Y \in \chi(M)$.

From (2.1) and (5.2), we get

$$
\left\{\begin{array}{l}
\Theta r=r \Theta=\theta r=\frac{\theta}{2 \sqrt{2}} \Theta+\frac{1}{2 \sqrt{2}} I, \\
\Theta s=s \Theta=(2-\theta) s=\frac{2-\theta}{2 \sqrt{2}} \Theta+\frac{1}{2 \sqrt{2}} I .
\end{array}\right.
$$

Then for silver structure, we can easily prove that

$$
\left\{\begin{array}{l}
s[r X, r Y]=\frac{1}{8} s N_{\Theta}(r X, r Y), \\
r[s X, s Y]=\frac{1}{8} r N_{\Theta}(s X, s Y) .
\end{array}\right.
$$

Proposition 5.1. A silver structure $\Theta$ is integrable if and only if the associated (2.4) almost product structure is integrable.

Proposition 5.2. Let $X, Y \in \chi(M)$. The distribution $R$ is integrable if and only if $s N_{\Theta}(r X, r Y)=0$, and the distribution $S$ is integrable if and only if $r N_{\Theta}(s X, s Y)=0$. If $\Theta$ is integrable then both the distributions $R$ an $S$ are integrable.

Let $\nabla$ be a linear connection on $M$. To the pair $(\Theta, \nabla)$ we associate two other linear connections $[1,3]$ :

i) The Schouten connection

$$
\tilde{\nabla}_{X} Y=r\left(\nabla_{X} r Y\right)+s\left(\nabla_{x} s Y\right)
$$

ii) The Vrănceanu connection

$$
\check{\nabla}_{X} Y=r\left(\nabla_{r X} r Y\right)+s\left(\nabla_{s x} s Y\right)+r[s X, r Y]+s[r X, s Y] .
$$

Proposition 5.3. The projectors $r$ and s are parallels in terms of Schouten and Vrănceanu connections for every linear connection $\nabla$ on $M$. Also, $\Theta$ is parallel in terms of Schouten and Vrănceanu connections.

Proof. From (5.1), for every $X, Y \in \chi(M)$

$$
\begin{gathered}
\left(\tilde{\nabla}_{X} r\right) Y=\tilde{\nabla}_{X} r Y-r\left(\tilde{\nabla}_{X} Y\right)=r\left(\nabla_{X} r Y\right)-r\left(\nabla_{X} r Y\right)=0 \\
\left(\check{\nabla}_{X} r\right) Y=\check{\nabla}_{X} r Y-r\left(\check{\nabla}_{X} Y\right)=r\left(\nabla_{r X} r Y\right)+r[s X, r Y]-r\left(\nabla_{r X} r Y\right)-r[s X, r Y]=0 .
\end{gathered}
$$

Thus, $r$ is parallel with respect to $\tilde{\nabla}$ and $\check{\nabla}$.

In a similar manner, it can be shown that $s$ is parallel with respect to $\tilde{\nabla}$ and $\check{\nabla}$. From (5.3), $\Theta$ is parallel with respect to Schouten and Vrănceanu connections. 
Definition 5.1 (see [5]). The distribution $R$ is called parallel with respect to linear connection $\nabla$ if $\nabla_{X} Y \in R$ where $X \in \chi(M)$ and $Y \in R$.

Definition 5.2 (see [5]). The distribution $R$ is called $\nabla$-half parallel if $(\Delta \Theta)(X, Y) \in R$ where

$$
(\Delta \Theta)(X, Y)=\Theta \nabla_{X} Y-\Theta \nabla_{Y} X-\nabla_{\Theta X} Y+\nabla_{Y}(\Theta X)
$$

for $X \in R, Y \in \chi(M)$.

Definition 5.3 (see [5]). The distribution $R$ is called $\nabla$-anti half parallel if $(\Delta \Theta)(X, Y) \in S$ where $X \in R$, $Y \in \chi(M)$.

Proposition 5.4. The distributions $R$ and $S$ are parallel in terms of Schouten and Vrănceanu connections for the linear connection $\nabla$.

Proof. Let $X \in \chi(M)$ and $Y \in R$. So, $s Y=0$ and $r Y=Y$. From (5.4) and (5.5), we get

$$
\begin{gathered}
\tilde{\nabla}_{X} Y=r\left(\nabla_{X} Y\right) \in R \\
\check{\nabla}_{X} Y=r\left(\nabla_{r X} Y\right)+r[s X, Y] \in R .
\end{gathered}
$$

Hence $R$ is parallel with respect to $\tilde{\nabla}$ and $\check{\nabla}$.

$S$ also satisfies similar equations.

Proposition 5.5. The distributions $R$ and $S$ are parallel with respect to $\nabla$ linear connection if and only if $\nabla$ and $\tilde{\nabla}$ are equal.

Proof. If $R, S$ are $\nabla$-parallel then $\nabla_{X}(r Y) \in R$ and $\nabla_{X}(s Y) \in S$ where $X, Y \in \chi(M)$.

For that reason

Since $r+s=I$ and (5.4),

$$
\nabla_{X}(r Y)=r \nabla_{X}(r Y) \text { and } \nabla_{X}(s Y)=s \nabla_{X}(s Y) .
$$

Therefore $\nabla=\tilde{\nabla}$.

$$
\nabla_{X} Y=r \nabla_{X}(r Y)+s \nabla_{X}(s Y)=\tilde{\nabla}_{X} Y
$$

The converse can be shown easily.

Proposition 5.6. The distribution $R$ is half parallel with respect to the Vrănceanu connection if

$$
[r X, s Y] \in R
$$

where $X \in R$ and $Y \in \chi(M)$.

Proof. Taking account of the equation (5.6) for connection $\check{\nabla}$, we have

$$
s(\Delta \Theta)(X, Y)=s \Theta \check{\nabla}_{X} Y-s \Theta \check{\nabla}_{Y} X-s \check{\nabla}_{\Theta X} Y+s \check{\nabla}_{Y}(\Theta X)
$$

where $X \in R$ and $Y \in \chi(M)$.

Hence, by (5.3) and (5.5), we obtain

$$
s(\Delta \Theta)(X, Y)=(2-2 \phi) s[r X, s Y]
$$

which proves the proposition.

Similarly, we have the following proposition for distribution $S$.

Proposition 5.7. The distribution $S$ is half parallel with respect to Vrănceanu connection if

$$
[s X, r Y] \in S
$$

where $X \in S$ and $Y \in \chi(M)$.

Proposition 5.8. The distributions $R$ and $S$ are anti half parallel with respect to Vrănceanu connection.

Proof. Taking account of the equation (5.6) for $\check{\nabla}$, we have

$$
r(\Delta \Theta)(X, Y)=r \Theta \check{\nabla}_{X} Y-r \Theta \check{\nabla}_{Y} X-r \check{\nabla}_{\Theta X} Y+r \check{\nabla}_{Y}(\Theta X)
$$

where $X \in R$ and $Y \in \chi(M)$.

From (5.3) and (5.5), we obtain

$$
r(\Delta \Theta)(X, Y)=(2 \phi-2) r[s X, r Y] .
$$

Since $s X=0$, we have $r(\Delta \Theta)(X, Y)=0$. Thus $(\Delta \Theta)(X, Y) \in S$.

In a similar manner, it can be shown that $S$ is anti half parallel with respect to the Vrănceanu connection. 


\section{Silver Riemannian Manifolds}

Let $P$ be almost product structure on manifold $M$ and $g$ be a Riemannian metric (respectively, a semiRiemannian metric) such as

$$
g(P(X), P(Y))=g(X, Y) \forall X, Y \in \chi(M)
$$

or equivalently, $P$ be a $g$-symmetric endomorphism such as

$$
g(P(X), Y)=g(X, P(Y)) .
$$

Then, we call that the pair $(g, P)$ is a Riemannian almost product structure (respectively, a semi-Riemannian almost product structure) $[8,27,31]$.

From (2.3) and (2.4), we can give the following proposition.

Proposition 6.1. The almost product structure $P$ is a g-symmetric endomorphism if and only if the associated silver structure $\Theta$ is also $g$-symmetric endomorphism.

Definition 6.1. Let $g$ be a Riemannian metric (respectively, a semi-Riemannian metric) on manifold $M$ such as

$$
g(\Theta(X), Y)=g(X, \Theta(Y)), \quad \forall X, Y \in \chi(M) .
$$

Then, we call that the pair $(g, \Theta)$ is a silver Riemannian structure (respectively, a silver semi-Riemannian structure) and triple $(M, g, \Theta)$ is also a silver Riemannian manifold (respectively, a silver semi-Riemannian manifold).

Corollary 6.1. Let $(M, g, \Theta)$ be a silver Riemannian manifold. Then, on a silver Riemannian manifold $(M, g, \Theta)$,

i) The projectors $r$, s are g-symmetric. That is,

$$
\left\{\begin{array}{l}
g(r(X), Y)=g(X, r(Y)) \\
g(s(X), Y)=g(X, s(Y))
\end{array}\right.
$$

ii) The distributions $R, S$ are g-orthogonal. That is,

$$
g(r(X), s(Y))=0
$$

iii) The silver structure $\Theta$ on manifold $M$ is $N_{\Theta}$-symmetric. That is,

$$
N_{\Theta}(\Theta(X), Y)=N_{\Theta}(X, \Theta(Y)) .
$$

Proposition 6.2 (see [3]). A Riemannian almost product structure is a locally product structure if $P$ is parallel with respect to the Levi-Civita connection $\stackrel{g}{\nabla}$ of $g$, i.e. $\stackrel{g}{\nabla} P=0$ and if $\nabla$ is a symmetric linear connection then the Nijenhuis tensor of $P$ verifies

$$
N_{P}(X, Y)=\left(\nabla_{P X} P\right) Y-\left(\nabla_{P Y} P\right) X-P\left(\nabla_{X} P\right) Y+P\left(\nabla_{Y} P\right) X .
$$

Then in the silver structure, we have

Proposition 6.3. The silver structure $\Theta$ is integrable if $(M, g, \Theta)$ is a locally product silver Riemannian manifold.

Theorem 6.1. The set of linear connections $\nabla$ for which $\nabla \Theta=0$ is

$$
\nabla_{X} Y=\frac{1}{4}\left[3 \bar{\nabla}_{X} Y+\Theta\left(\bar{\nabla}_{X} \Theta Y\right)-\Theta\left(\bar{\nabla}_{X} Y\right)-\bar{\nabla}_{X} \Theta Y\right]+O_{p} Q(X, Y)
$$

where $\bar{\nabla}$ is an arbitrary linear connection and $Q$ is a $(1,2)$-tensor field for which $O_{P} Q$ is an associated Obata operator

$$
O_{P} Q(X, Y)=\frac{1}{2}[Q(X, Y)+P Q(X, P Y)]
$$

for the corresponding almost product structure (2.3).

Now, let us give the following example for silver structure. 


\section{Example 6.1.}

$$
\begin{aligned}
& r=\frac{1}{(x+y)^{2}+1} \frac{\partial}{\partial x} \otimes d x-\frac{x+y}{(x+y)^{2}+1} \frac{\partial}{\partial x} \otimes d y-\frac{x+y}{(x+y)^{2}+1} \frac{\partial}{\partial y} \otimes d x+\frac{(x+y)^{2}}{(x+y)^{2}+1} \frac{\partial}{\partial y} \otimes d y \\
& s=\frac{(x+y)^{2}}{(x+y)^{2}+1} \frac{\partial}{\partial x} \otimes d x+\frac{x+y}{(x+y)^{2}+1} \frac{\partial}{\partial x} \otimes d y+\frac{x+y}{(x+y)^{2}+1} \frac{\partial}{\partial y} \otimes d x+\frac{1}{(x+y)^{2}+1} \frac{\partial}{\partial y} \otimes d y
\end{aligned}
$$

are projection operators in $\mathbb{R}^{2}$.

$$
R=S p\left\{\frac{\partial}{\partial x}-(x+y) \frac{\partial}{\partial y}\right\} \quad \text { and } \quad S=S p\left\{(x+y) \frac{\partial}{\partial x}+\frac{\partial}{\partial y}\right\}
$$

are complementary distributions corresponding to the projection operators $r$ and $s$, respectively. The distributions $R, S$ are orthogonal with respect to the Euclidean metric of $\mathbb{R}^{2}$. Moreover, these distributions are associated to the silver structure

$$
\begin{aligned}
\Theta\left(\frac{\partial}{\partial x}\right) & =\frac{(2-\theta)(x+y)^{2}+\theta}{(x+y)^{2}+1} \frac{\partial}{\partial x}-\frac{2 \sqrt{2}(x+y)}{(x+y)^{2}+1} \frac{\partial}{\partial y} \\
\Theta\left(\frac{\partial}{\partial y}\right) & =-\frac{2 \sqrt{2}(x+y)}{(x+y)^{2}+1} \frac{\partial}{\partial x}+\frac{\theta(x+y)^{2}+(2-\theta)}{(x+y)^{2}+1} \frac{\partial}{\partial y}
\end{aligned}
$$

which is integrable since $N_{\Theta}\left(\frac{\partial}{\partial x}, \frac{\partial}{\partial y}\right)=0$.

\section{Acknowledgments}

The authors would like to thank the referees for their valuable comments and suggestions to improve the quality of the paper.

\section{References}

[1] Bejancu, A. and Farran, H.R., Foliations and Geometric Structures. Mathematics and its Applications, vol. 580, Springer, 2006.

[2] Chandra, M. and Rani, M., Categorization of fractal plants. Chaos, Solitons \& Fractals 41 (2009), no.3, 1442-1447.

[3] Crasmareanu, M. and Hrețcanu, C.E., Golden differential geometry. Chaos, Solitons \& Fractals 38 (2008), no.5, 1229-1238.

[4] Cruceanu, V., On almost biproduct complex manifolds. An. Ştiinţ. Univ. Al. I. Cuza Iaşi. Mat. (N.S.) 52 (2006), no.1, 5-24.

[5] Das, L.S., Nikic, J. and Nivas, R., Parallelism of distributions and geodesics on $F\left(a_{1}, a_{2}, \ldots, a_{n}\right)$-structure Lagrangian manifolds. Diff. Geom. Dyn. Syst. 8 (2006), 82-89.

[6] Gezer, A., Cengiz, N. and Salimov, A., On integrability of Golden Riemannian structures. Turk. J. Math. 37 (2013), no.4, $693-703$.

[7] Gezer, A. and Karaman C., Golden-Hessian structures. Proc. Natl. Acad. Sci., India, Sect. A Phys. Sci. 86 (2016), no:1, 41-46.

[8] Gray, A., Pseudo-Riemannian almost product manifolds and submersions. J. Math. Mech. 16 (1967), 715-737.

[9] Hinterleitner, I., Mikeš, J. and Peška, P., On $F_{2}^{\varepsilon}$-planar mappings of (pseudo-) Riemannian manifolds. Arch. Math. (Brno) 50 (2014), no.5, 287-295.

[10] Hrdina, J., Geometry of almost Cliffordian manifolds: Nijenhuis tensor. Miskolc Math. Notes 14 (2013), no.2, 583-589.

[11] Hrdina, J. and Vašik, P., Geometry of almost Cliffordian manifolds: classes of subordinated connections. Turk. J. Math. 38 (2014), no.1, 179-190.

[12] Horadam, A.F., Pell identities. Fibonacci Quart. 9 (1971), no.3, 245-252, 263.

[13] Hretcanu, C.E., Submanifolds in Riemannian manifold with Golden structure. Workshop on Finsler geometry and its applications, Hungary, 2007.

[14] Hrețcanu, C.E. and Crasmareanu, M., On some invariant submanifolds in a Riemannian manifold with Golden structure. An. Ştiinţ. Univ. Al. I. Cuza Iaşi. Mat. (N.S.) 53 (2007), suppl. 1, 199-211.

[15] Hrețcanu, C.E. and Crasmareanu, M., Applications of the Golden ratio on Riemannian manifolds. Turk J. Math. 33 (2009), no.2, $179-191$.

[16] Kocer, E.G., Tuglu, N. and Stakhov, A., Hyperbolic functions with second order recurrence sequences. ARS Combinatoria 88 (2008), 65-81.

[17] Mikeš, J., et al., Differential Geometry of Special Mappings. Palacký University, Faculty of Science, Olomouc, 2015.

[18] Mikeš, J., Jukl, M. and Juklovǎ, L., Some results on traceless decompositon of tensors. J. Math. Sci. 174 (2011), no.5, 627-640.

[19] Mikeš, J. and Sinyukov, N.S., On quasiplanar mappings of spaces of affine connection. Sov. Math. 27 (1983), 63-70.

[20] Primo, A. and Reyes, E., Some algebraic and geometric properties of the silver number. Mathematics and Informatics Quarterly 18 (2007), no. $\frac{1}{2}$.

[21] Procesi, C., Lie Groups: An Approach Through Invariants and Representations. Universitext, Springer, 2007.

[22] Özdemir, F. and Crasmareanu, M., Geometrical objects associated to a substructure. Turk J. Math. 35 (2011), no.4, 717-728.

[23] Özkan, M. and Peltek, B., Silver differential geometry. II. International Eurasian Conference on Mathematical Sciences and Applications, Sarajevo-Bosnia and Herzegovina, 2013, 273. 
[24] Özkan, M., Prolongations of Golden structures to tangent bundles. Differ. Geom. Dyn. Syst. 16 (2014), $227-238$.

[25] Özkan, M., Çıtlak, A.A. and Taylan, E., Prolongations of Golden structure to tangent bundle of order 2. GU J. Sci. 28 (2015), no.2, 253-258.

[26] Özkan, M. and Y1lmaz, F., Prolongations of Golden structures to tangent bundles of order r. Commun. Fac. Sci. Univ. Ank. Ser. A1 Math. Stat. 65 (2016), no.1, 35-47.

[27] Pripoae, G.T., Classification of semi-Riemannian almost product structure. Proceedings of The Conference of Applied Differential Geometry - General Relativity and the Workshop on Global Analysis, Differential Geometry and Lie Algebras, 2002, 243-251.

[28] Savas, M., Ozkan, M. and Iscan, M., On 4-dimensional Golden-Walker structures, Journal of Science and Arts, (2016), no.2(35), 89-100.

[29] Şahin, B. and Akyol, M.A., Golden maps between Golden Riemannian manifolds and constancy of certain maps. Math. Commun. 19 (2014), no. 2, 333-342.

[30] Yano, K. and Ishihara, S., Tangent and Cotangent Bundle. Marcel Dekker Inc., New York, 1973.

[31] Yano, K. and Kon, M., Structures on Manifolds, Series in Pure Mathematics. Vol. 3, World Scientific, Singapore, 1984.

[32] Yardımc1, E.H. and Yaylı, Y., Golden quaternionic structures. Int. Electron. J. Pure Appl. Math. 7 (2014), no.3, 109-125.

\section{Affiliations}

MUSTAFA OZKAN

AdDress: Gazi University, Faculty of Science, Dept. of Mathematics, 06500, Ankara-Turkey.

E-MAIL: ozkanm@gazi.edu.tr

BETUl PELTEK

AdDress: Gazi University, Faculty of Science, Dept. of Mathematics, 06500, Ankara-Turkey.

E-MAIL: betul-p@hotmail.com 